\title{
Lokale Gerechtigkeit und Governanceethik
}

\section{ALEXANDER BRINK ${ }^{* *}$}

\section{Local Justice and Governance Ethics}

This paper will take insights from John Elster's Theory of Local Justice and attempt to display their fruitfulness for business ethics. The paper begins with a reconstruction of its content, argumentation, and method. Basically, the theory of local justice deals with decisions of rationalization of decentralized organizations. In his theory, Elster touches an empirical-normative gray area. Essentially, his theory seeks the connection of normative theories of justice with empirical practice as well as a normative reflection upon the empirical conditions of modern societies. Based on Elster's rational-choice approach the theory of local justice is extended to business etbics by employing three perspectives: justification theory, institutional economics and contract theory. Wieland's etbics of governance integrates these three perspectives of local justice. Governance refers to an adaptive characteristic: the ability of steering processes to adapt to specific local transactions. Thus the local mechanism of governance itself provides the systematic location of justice.

Keywords: Jon Elster, Local Justice, Philosophy of Science, Business Ethics

\section{Einleitung}

John Rawls schreibt in seiner Theorie der Gerechtigkeit: „[D]enn was gerecht oder ungerecht sei, ist gewöhnlich umstritten“" (Rawls 1971: 21). Die begriffliche Vielfalt und Unbestimmtheit des Gerechtigkeitsbegriffs wird in dem für die interdisziplinäre Gerechtigkeitsforschung bedeutsamen Buch Local Justice von Jon Elster zum Programm (vgl. Elster 1992). Es geht bei der Theorie lokaler Gerechtigkeit um die Verteilung von knappen und unteilbaren Gütern und Lasten, die gerechtigkeitstheoretisch in den Bereich der Institutionen fällt, genauer: in die institutionellen Sektoren einer Gesellschaft (vgl. Elster 1990, 1992). Sie ist deshalb lokal, weil die Verteilungsentscheidung sachlich, räumlich, zeitlich und sozial begrenzt ist: „different institutional sectors use different substantive principles of allocations" (Elster 1992: 3). ${ }^{1}$ Im Gegensatz zur

\footnotetext{
* $\quad$ Beitrag eingereicht am 06.05.2010, nach doppelt verdecktem Gutachterverfahren überarbeitete Fassung angenommen am 19.01.2011.

** Prof. Dr. Dr. Alexander Brink, Professor für Wirtschafts- und Unternehmensethik an der Universität Bayreuth, Institut für Philosophie, Universitätsstraße 30, D-95440 Bayreuth, Tel: +49 (0)921/554122, Fax: +49 (0)921/554160, Email: alexander.brink@uni-bayreuth.de. Permanenter Gastprofessor für Corporate Governance \& Philosophy am Reinhard-Mohn-Institut der Privaten Universität Witten/Herdecke, Alfred-Herrhausen-Straße 50, D-58448 Witten, Tel: +49 (0)2302/ 926572, Tel: +49 (0)2302/926587, Email: alexander.brink@uni-wh.de. Forschungsschwerpunkte: Corporate Governance, Corporate Social Responsibility, NPO-Forschung, Neue Institutionenökonomik und Vertragstheorie.

$1 \quad$ Vgl. zur lokalen Gerechtigkeit auch Walzer (1992) und Schmidt (1995: 177ff.).
} 
global justice wird bei der lokalen Gerechtigkeit die Entscheidung dezentral getroffen, es erfolgt keine Kompensation für Benachteiligte und es wird in der Regel keine Geld-, sondern eine Sachleistung erbracht (vgl. Elster 1991: 274; Elster 1992: 4 und Elster 1995). Die mangelhafte Berücksichtigung solcher eher soziologischer Erkenntnisse in der Gerechtigkeitsforschung wird von zahlreichen Autoren angemahnt (vgl. Schmidt 1995 und 2000; Müller 1992 und Müller/Wegener 1995): „Die Soziologie hat, folgt man dem Urteil informierter Chronisten, zu dieser Frage erstaunlich wenig zu sagen“ (Schmidt 2000: 9).

Im Zentrum des vorliegenden Beitrags steht der Versuch, die Theorie lokaler Gerechtigkeit von Jon Elster für die Wirtschafts- und Unternehmensethik fruchtbar zu machen. Zunächst werden Inhalt, Argumentationsgang und Methode nachgezeichnet (Kapitel 2). Elster bewegt sich in einer empirisch-normativen Grauzone, was Konsequenzen für sein Verständnis von Ethik hat. Es geht ihm im Ergebnis um die Anbindung normativer Gerechtigkeitstheorien an die empirische Praxis oder umgekehrt um die normative Reflexion empirischer Bedingungen moderner Gesellschaften (Kapitel 3). Ausgehend von Elsters Rational-Choice-Ansatz wird die Theorie lokaler Gerechtigkeit hinsichtlich drei Perspektiven wirtschafts- und unternehmensethisch erweitert: begründungstheoretisch, institutionenökonomisch und vertragstheoretisch (Kapitel 4). In Wielands Governanceethik werden diese drei perspektivischen Erweiterungen lokaler Gerechtigkeit aufgegriffen. Im Rahmen seiner Governanceethik wird die lokale Gerechtigkeit soziologisch an die Wirtschafts- und Unternehmensethik angeschlossen. Governance bezeichnet dabei die Adaptivität, d. h. die Anpassungsfähigkeit von Steuerungsstrukturen an konkrete lokale Transaktionen: Der lokale Governancemechanismus selbst wird zum systematischen Ort der Gerechtigkeit (Kapitel 5).

\section{Elsters Theorie lokaler Gerechtigkeit}

Elster stellt seine Theorie der lokalen Gerechtigkeit in drei sich inhaltlich überschneidenden Werken vor: Local Justice - How Institutions Allocate Scarce Goods and Necessary Burdens (vgl. Elster 1992), Ethique des Choix Médicaux (vgl. Elster/Herpin 1992) und Local Justice in America (vgl. Elster 1995). Im Folgenden möchte ich daraus einige wesentliche Bausteine seiner Theorie skizzieren, sofern sie für das hier zu entfaltende Argument zielführend sind. ${ }^{2}$ Die Theorie der lokalen Gerechtigkeit befasst sich grundlegend mit Rationierungsentscheidungen dezentraler Organisationen. Untersuchungsgegenstand sind die Prinzipien und deren Anreize für die Allokation knapper Zuteilungsgüter in der Praxis. ${ }^{3}$ Elster formuliert das folgendermaßen: Schmidt (1992a/b, 1995, 2000).

3 Zuteilungsgüter sind solche Güter, bei denen eine Leistung ohne direkte Gegenleistung erfolgt. Die Verteilung (allocation) lässt sich nach dem Vorhandensein bzw. Nicht-Vorhandensein der folgenden Kriterien einordnen: (1) Knappheit (scarcity), (2) Unteilbarkeit (indivisibility) und (3) Homogenität (Gleichheit) (vgl. Elster 1992: 21ff.). Probleme der local justice im eigentlichen Sinne (core cases of local justice bzw. local justice stricto sensu) setzen Knappheit und Heterogenität voraus (Ausnahmen einer Verteilung von nicht-knappen und homogenen Gütern sind hingegen etwa Stimmrechte) (vgl. Elster 1992: 26). 
„For a given good $\mathrm{A}$, is there any principle $\mathrm{X}$ that is never used to allocate it? For a given principle $\mathrm{X}$, is there any good $\mathrm{A}$ such that $\mathrm{X}$ is never used to allocate A? Are there pairs $(\mathrm{A}, \mathrm{X})$ such that $\mathrm{A}$ is always allocated by $\mathrm{X}$ ? Are there patterns of covariation, such that if a society uses principle $\mathrm{X}$ to allocate good $\mathrm{A}$ it will use principle Y to allocate good B?“" (Elster 1992: 15).

Die Anwendungsbereiche lokaler Gerechtigkeit sind vielfältig und reichen von Nierentransplantationen, Universitätsaufnahmen bis hin zu Mitarbeiterentlassungen (vgl. Elster 1992: 1 und 28ff.). Zwar wird vieles davon durch den Markt bzw. durch die Politik geregelt ${ }^{4}$ - oftmals sind es aber die bewussten Entscheidungen quasi autonomer Institutionen wie z. B. bei den Entfaltungsmöglichkeiten von individuellen Lebenschancen (vgl. Elster 1992: 2). Es sind autonome Allokationsverfahren wie Auswahl (selection), Zulassung (admission) und Vergabe (placement), die Elster besonders interessieren (vgl. Hofstee 1990 und Elster 1992: 24ff.). Obwohl Gerechtigkeits- und Verteilungsfragen vereinzelt in der Ökonomie bzw. in den Politikwissenschaften behandelt werden, fehlt bislang - so Elster - eine umfassende Analyse:

„[T] here have been virtually no attempts to study the whole range of questions of this kind, and to develop a conceptual and theoretical framework to describe and explain how institutions allocate goods and burdens" (Elster 1992: 2).

Diesem von Elster bislang als puzұling bezeichneten Phänomen möchte der Soziologe nunmehr systematisch auf den Grund gehen. Damit ist sein Forschungsprogramm definiert. Elsters Anspruch ist keineswegs die Entwicklung einer eigenen Theorie. Es sind die sozialen Mechanismen (social mechanisms), die eine Verbindung zwischen der Theorie auf der einen und der Beschreibung von Wirklichkeit auf der anderen Seite herstellen (vgl. Elster 1992: 14ff. und 135ff.). Ein sozialer Mechanismus wird definiert als „,identifiable causal pattern that comes into play under certain, generally unknown, conditions" (Elster 1992: 15). Damit können sich soziale Mechanismen im Laufe der Zeit verändern (Elster 1992: 137), so dass Lösungen für bestimmte Probleme keineswegs dauerhaft erreicht werden. Im Zentrum steht also die Adaption der Mechanismen durch die Lokalität von Gerechtigkeit. ${ }^{5}$

Nach welchen Prinzipien lokaler Gerechtigkeit werden nun aber diese Entscheidungen getroffen? Elster verweist auf eine Liste von Zuteilungskriterien, die so genannten „elementary building blocks of local justice“ (Elster 1992: 103), nach denen dezentrale Organisationen über Probleme lokaler Gerechtigkeit in vielen Fällen entscheiden. Diese Liste von Zuteilungskriterien umfasst aber lediglich „,all the major principles“ (Elster 1992: 62). Es ist keine Typologie „based on first principles that would generate exhaustive and mutually exclusive categories“ (Elster 1992: 62). So gibt es z. B. Prinzipien, die sich auf bestimmte individuelle Eigenschaften beziehen (z. B., dass jeder

$4 \quad$ Gerade den Markt will Elster aber bewusst auslassen: „Allocations that emerge as the result of decentralized, uncoordinated decisions - the market being the paradigm case - have no place in the present classification" (Elster 1992: 68). Eine Ausnahme bildet der Fall, bei dem eine Institution Markmechanismen bewusst einführt.

$5 \quad$ Vgl. auch die governanceethische Weiterentwicklung, bei der dieses Argument aufgenommen wird: Auch hier steht die Adaption von Governancemechanismen im Zentrum (vgl. Kapitel 5). 
gemäß seinen Bedürfnissen bedient werden soll). Andere Prinzipien sind in Mechanismen (mechanisms) eingebunden, welche keinerlei Kenntnis über den Empfänger enthalten wie etwa Lotterien (lotteries). Insgesamt lassen sich fünf, miteinander kombinierbare Kategorien unterscheiden, die Elster ausführlich erläutert und mit zahlreichen Beispielen unterlegt. Die folgende Tabelle fasst diese Kategorien zusammen (vgl. Elster 1992: 70ff.):

\begin{tabular}{|c|c|}
\hline $\begin{array}{c}\text { Gruppe von Prinzipien lokaler Gerechtigkeit } \\
\text { (building blocks of local justice) }\end{array}$ & $\begin{array}{c}\text { Auswahl von Prinzipien } \\
\text { (selection of principles) }\end{array}$ \\
\hline $\begin{array}{c}\text { Egalitäre Kriterien } \\
\text { (egalitarian principles) }\end{array}$ & $\begin{array}{c}\text { absolute Gleichheit (absolute equality), Lotterien } \\
\text { (lotteries), gleiche Abweichung von einer Grundlinie } \\
\text { (equal deviation from a baseline) und Rotationsverfahren } \\
\text { (rotation). }\end{array}$ \\
\hline $\begin{array}{c}\text { Zeitbasierte Kriterien } \\
\text { (time-related principles) }\end{array}$ & $\begin{array}{c}\text { Warteschlangen (queuing), Wartelisten (waiting lists) } \\
\text { und Senioritätsprinzip (seniority). }\end{array}$ \\
\hline $\begin{array}{c}\text { Statusbasierte Kriterien } \\
\text { (principles defined by status) }\end{array}$ & $\begin{array}{c}\text { Alter (age), Geschlecht (gender), sexuelle Orientierung } \\
\text { (sexual orientation), Wohnort (residence status), Beruf } \\
\text { (occupational status), andere körperliche Merkmale } \\
\text { (other physical features) etc. }\end{array}$ \\
\hline $\begin{array}{c}\text { Situationsbasierte Kriterien } \\
\text { (principles defined by other properties) }\end{array}$ & $\begin{array}{c}\text { Individueller Wohlstandslevel (individual levels of } \\
\text { welfare), Bedürftigkeit (need), individueller Wohl- } \\
\text { fahrtszuwachs (individual increments of welfare), (lo- } \\
\text { kale) Effizienz (llocal] efficiency), Leistung (contribu- } \\
\text { tion), Charakter (character) }\end{array}$ \\
\hline $\begin{array}{c}\text { Machtbasierte Mechanismen } \\
\text { (mechanisms based on power) }\end{array}$ & $\begin{array}{c}\text { Kaufkraft (purchasing power), Einfluss (influence) } \\
\text { Kombinationen aus (1) bis (5) } \\
\text { (mixed principles) }\end{array}$ \\
$\begin{array}{c}\text { Simultane Berücksichtigung verschiedener Kriterien } \\
\text { (Punktbewertungssysteme), sukzessive Berücksichti- } \\
\text { gung verschiedener Kriterien, Verbindung von unper- } \\
\text { sönlichen Mechanismen (impersonal mechanisms) und } \\
\text { individuellen Kriterien (individualized criteria) }\end{array}$ \\
\hline
\end{tabular}

Abbildung 1: Gruppe von Prinzipien lokaler Gerechtigkeit (eigene Darstellung)

Nicht jede Allokation knapper Güter wird über diese Prinzipien gesteuert: Es gibt Fälle, in denen Institutionen diskretionär entscheiden. ${ }^{6}$

Neben den Prinzipien, die für Elster von zentraler Bedeutung sind, werden die Wirkungen oder Konsequenzen der lokalen Gerechtigkeit untersucht, die nach Primär- und Sekundäreffekten unterschieden werden. ${ }^{7}$ Sie werden vom Entscheider - bei Elster ist das der allocator bzw. der rule maker - vor der Wahl der Entscheidungsgrundlage antizi-

6 In Anlehnung an Selten werden darüber hinaus procedures (standards of distribution) von den principles (standards of comparison) unterschieden (vgl. Selten 1978). Schließlich werden schemes eingeführt, die als „full set of procedures used to implement mixed principles“ (Elster 1992: 63) bezeichnet werden.

$7 \quad$ Zu unterscheiden sind „secondary effects and incentive effects that obviously have no explanatory role, as well as those that have clear or possible explanatory import“" (Elster 1992: 114). 
piert. Dadurch können unter dem Deckmantel lokaler Gerechtigkeit Ausstrahlungseffekte bewusst in Kauf genommen, in einigen Fällen sogar absichtlich bewirkt und damit bestimmte Gruppen indirekt diskriminiert werden. Elster untersucht vor allem solche Sekundär- bzw. Ausstrablungseffekte, die Ungleichbehandlungen bewirken können. Anhand von Beispielen zeigt er, welche lokalen Gerechtigkeitsprinzipien Schwarze in den USA von Nierentransplantationen ausgeschlossen haben (vgl. Elster 1992: 115ff.) oder Europäern die Einwanderung in die USA erschwerten (vgl. Elster 1992: $121 \mathrm{ff}$.). In einem anderen prominenten Beispiel wurden Juden bei der Aufnahme an der Universität Yale indirekt diskriminiert (vgl. Elster 1992: 117ff.). In Erinnerung an einen Skandal in Harvard aus dem Jahre 1923, hervorgerufen durch eine Quotierung für jüdische Studierende, erfolgte in Yale im vorliegenden Fall eine indirekte Diskriminierung: „Officially, Yale never had quotas for Jews“ (Elster 1992: 117). Durch die Einführung eines Auswahlprinzips, das die geographical diversity in den Blick nahm, erzielte man dennoch eine indirekte Diskriminierung:

„Though many individual Jews (concentrated in the northeast region from which Yale received most of its applications) would be affected by this principle, it was not an innately anti-Jewish principle" (Elster 1992: 118).

Auf Seiten potentieller Adressaten können ebenfalls Anpassungsvorgänge eingeleitet werden, sofern diese die Regeln nicht nur kennen, sondern auch verstehen und in der Lage sind, ihr Verhalten entsprechend zu ändern. ${ }^{8}$ Ein bekannter Anreizeffekt ist das moral hazard (vgl. Elster 1992: 125ff.). ${ }^{9}$ Nach Vertragsabschluss - hier in Form der Gültigkeit eines bestimmten Prinzips - wird der Empfänger versuchen, in den Genuss des Gutes zu kommen, und sein Verhalten entsprechend anpassen: So besteht z. B. nur ein geringer Anreiz erfolgreich zu arbeiten, wenn eine Vergütung gemäß dem Senioritätsprinzip (seniority) erfolgt (vgl. Elster 1992: 125 und Elster 1991: 286).

Obwohl Elsters Theorie die lokale Gerechtigkeit umfasst, interessiert ihn das Verhältnis zur globalen Gerechtigkeit. ${ }^{10}$ Es kann nämlich sein, dass „the search for local justice may produce global injustice“ (Elster 1991: 291). Hier kommt die Verteilungsgerechtigkeit (distributive justice) ins Spiel. Sie soll Ungleichheiten, die sich aus den Folgen dezentraler Märkte ergeben, ausgleichen (vgl. Elster 1992: 132f.). Gleichzeitig produziert sie neue Ungleichheiten - allerdings:

„I do believe that sometimes the total effect of local decisions is to create a pattern of burdens and benefits that seems unfair, inefficient, or both. The importance of such effects may be more than marginal, but certainly less than pervasive“ (Elster 1992: 132).

Lotterien etwa haben keinen Anreizeffekt, weil man sie qua definitionem nicht beeinflussen kann.

Vgl. zur institutionenökonomischen Erweiterung Kapitel 4.3.

Es sei an dieser Stelle darauf verwiesen, dass Elster unter der global justice keine universalistische Prinzipiengerechtigkeit versteht, wie man sie etwa bei John Rawls findet (vgl. Rawls 1971), sondern einen ,institutionenunabhängige[n] gerechtigkeitstheoretische[n] Konsens unter den gesellschaftlich führenden Entscheidungsträgern" (Wegener 1995: 258). 
Es ergibt sich ein weiteres Problem, auf das Elster hinweist: So mögen Entscheidungen durch Institutionen über weite Strecken hinweg als gerecht empfunden werden. Das Individuum jedoch „encounters a succession of institutions" (Elster 1992: 133) und kann die Summe der von Institutionen gefällten Entscheidungen für sich als unfair empfinden. Diese Form von Ungerechtigkeit - so Elster - sei bislang noch nicht hinlänglich untersucht worden, da die Entscheidungen lokaler Gerechtigkeiten nie im Lichte der individuellen Erfahrungen des Einzelnen gesehen wurden: „Local justice is largely noncompensatory. There is no mechanism of redress across allocative spheres" (Elster 1992: 133). Gerechtigkeit wird also dadurch bestimmt, inwieweit die prinzipienorientierte Entscheidung einer Institution individuelle Präferenzen in lokalen Entscheidungssituationen in der Summe erfüllt oder enttäuscht.

Elsters Theorie hat nicht nur beschreibende Teile, sondern erklärt auch, „why, at particular time and place, a particular institution adopts a particular principle for allocating a particular good" (Elster 1992: 135 sowie ähnlich Elster 1991: 286). In Anlehnung an Calabresi/Bobbit unterscheidet er drei Entscheidungsebenen (three levels of decision making) (vgl. Elster 1992: 139ff. und Calabresi/Bobbit 1978): First-order decisions umfassen sämtliche zu treffende Entscheidungen, um die Gesamtmenge eines Gutes zu verteilen, die second-order decisions beziehen sich auf die Frage, über welche Prinzipien die Allokation erfolgt. Third-order decisions beziehen sich schließlich auf die Empfänger und deren Bedürfnis, ein Gut nachzufragen, bzw. deren Wahrscheinlichkeit, es zu erhalten, und zwar unter einer entsprechenden Anreizstruktur. ${ }^{11}$ Entsprechend werden auch drei Akteursgruppen differenziert (vgl. Elster 1992: 140ff.): Primärakteure (Akteure erster Ordnung) werden als politische Akteure (political authorities) bezeichnet, die den Rahmen, die Bedingungen und damit auch die Prinzipien festlegen, nach denen Sekundärakteure den Tertiärakteuren Güter zuweisen. Sie können Anreize setzen bzw. Anpassungsprozesse von Tertiärakteuren antizipieren. Sekundärakteure (Akteure zweiter Ordnung) umfassen die Zuteilungsorganisationen bzw. deren Entscheider (allocators), also inner-institutionelle Akteure wie Beamte oder Personalverantwortliche. ${ }^{12}$ Die Sekundärakteure sind für Elster die wichtigsten Akteure. Tertiärakteure (Akteure dritter Ordnung) sind potentielle Nachfrager, Empfänger oder Antragsteller (recipients) sowie die von Ausstrahlungseffekten betroffenen Bürger. ${ }^{13}$

11 Calabresi/Bobbits Zwei-Ebenen-Modell folgend, betrifft die first-order decision die Entscheidung über die Menge an Gütern, die es zu verteilen gilt, und die second-order decision befasst sich mit der Art des Verteilungsprinrips. Elster ändert die first-order decisions leicht ab, übernimmt die second-order decisions und führt eine dritte Kategorie, die third-order decisions, ein (vgl. Elster 1992: 139).

12 Elster beschreibt in diesem Zusammenhang verschiedene Faktoren, die für die Präferenzbildung (preference formation) entscheidend sind: u. a. Strukturvariablen (structural variables), Kultur (national culture), Interessengruppen (organized interest groups), Anreizprobleme (incentive problems) oder etwa Informationsprobleme (information problems) (vgl. Elster 1992: 143ff.).

13 Ausführlich befasst Elster sich mit der public opinion an späterer Stelle (vgl. Elster 1992: 155ff.). Kritisch könnte man anmerken, dass Elster keine Akteure vierter Ordnung erwähnt. So wäre die Öffentlichkeit bzw. die öffentliche Meinung (general public) als weiterer Akteur zu benennen, auf die Elster an verschiedenen Stellen implizit aufmerksam macht: „Rule makers are to some extent also constrained by public opinion“" (Elster 1995: 20) bzw. ,public opinion can be seen as a quasi-actor" (Elster 1992: 143). 
Schließlich unterscheidet Elster drei Motivtypen (types of motives): Eigeninteresse (selfinterest), Gleichheit (equity) und Effizienz (efficiency) (vgl. Elster 1992: 180ff.). Die drei Motivationstypen lassen sich mit den drei Akteursgruppen - also Primär-, Sekundär-, Tertiärakteuren - kombinieren. Primärakteure sind überwiegend effizienzmotiviert, aufgrund des öffentlichen Drucks auch gleichheitsmotiviert. ${ }^{14}$ Sekundärakteure erhalten ihrerseits Motivation aufgrund der Gleichheit und der Effizienz, letztere ist allerdings eher lokal geprägt. Und schließlich sind die Tertiärakteure v. a. selbstinteressiert. Aufgrund der verschiedenen Präferenzen können sich nunmehr bestimmte Koalitionen bilden oder aber es entstehen spezifische Verhandlungssituationen. Skandale, die durch die Öffentlichkeit rezipiert werden, sind vermeidbar, sofern man von der Ergebnis- zur Verfahrensgerechtigkeit wechselt. Dies geschieht nach Elster durch den Wandel weg von diskretionären Entscheidungsregeln hin zu robusten - also klaren, transparenten und damit nicht-manipulierbaren - Entscheidungsregeln.

\section{Elsters Methodik zwischen Empirie und Normativität}

Elster versteht unter Gerechtigkeit „the allocation of scarce goods for the purpose of maximizing some aggregate features of the recipients or, more generally, of all citizens“ (Elster 1992: 6). Diese Vorgehensweise bezeichnen einige Autoren ,als empirisch (...), insbesondere nicht normativ" (Pies/Viebranz 2008: 181). Die Phänomenologie der lokalen Gerechtigkeit erfolgt - wie im zweiten Kapitel gezeigt wurde - über eine Typologisierung seiner Forschungsergebnisse. In seinem Buch Local Justice sei das Ziel einer vollständigen Phänomenologie nahezu erreicht: „The list, again, is long and this time is intended to be roughly exhaustive" (Elster 1992: 16 und ähnlich Elster 1992: 62). ${ }^{15}$ Elster wendet sich gegen einen normativen Universalismus und damit gegen die Vorstellung, es könne ein vollständiges Set von Gerechtigkeitsprinzipien oder sogar nur ein einziges Gerechtigkeitsprinzip geben, welches handlungsleitend für den Akteur sei (vgl. Wegener 1995: 256 und Schmidt 1995). Diese Auffassung wird unter anderem dadurch begründet, dass in modernen Gesellschaften Entscheidungen über die Verteilung von knappen Gütern oftmals in einzelnen Institutionen getroffen werden und es damit unmöglich sei, ein einziges koordinierendes Gerechtigkeitsprinzip zu bilden. Am Ende des Buches Local Justice bestärkt Elster diesen Eindruck,

„since these ideas are grounded in the practice of allocative decision making rather than in intuitions about hypothetical cases, their empirical foundations might be more robust than those of philosophical theories“ (Elster 1992: 237).

Deutlich formuliert er an einer anderen Stelle, ,[t]he notion of justice ${ }^{6}$ is used here mainly for explanatory rather than normative purposes“ (Elster 1992: 4), und verweist

14 Selbstinteresse spielt bei den Primärakteuren nur eine geringe Rolle, da knappe Güter über Verbindungen oder über den Wohlstand der Primärakteure in der Regel einfach zu erhalten sind (vgl. Elster 1992: 181).

15 Elster zeigt eine breite Kenntnis der Literatur (buman sciences und philosophy), mit Hilfe derer er seine Aussagen empirisch untermauert (vgl. Moon 1994: 180). Dabei fällt auf, dass unterschiedliche Verteilungsprobleme auf ähnlichen Prinzipien basieren (vgl. Mellers 1997). 
auf Walzers Spheres of Justice als normative Alternative: „Walzer's focus is mainly normative“" (Elster 1992: 11; vgl. auch Elster 1992: 9 und Walzer 1992). Walzer vertritt eine

„allgemeine Vorstellung von einer Eigenständigkeit von Gerechtigkeitsregeln und von der Autonomie einzelner Verteilungssphären (...). Keine einzelne sachliche Verteilungsregel kann Allgemein-Zuständigkeit beanspruchen, aber dennoch gibt es eine universelle Verfahrensregel: Jedes Gut soll nach den Geltungskriterien seiner eigenen ,Sphäre‘ zugeteilt werden“" (Walzer 1992: 12). ${ }^{16}$

Elster ist aber auch „kritisch“ gegenüber der empirischen Forschung. Im Gegensatz zu den gemeinsamen Ergebnissen von Ökonomen und Psychologen grenzt er sich z. B. von der Literatur zur experimentellen Gerechtigkeitsforschung ab und bezeichnet diese als „long on techniques, short on ideas“ (Elster 1992: 192). Moon etwa schreibt: „Although deeply concerned with normative issues [sic!], the principal focus of the work is descriptive and explanatory" (Moon 1994: 180). Empirisch wohlformulierte Entscheidungsprinzipien sind offensichtlich - und entgegen Elsters Intention (vgl. Elster 1992: 4) - nicht von den normativen Aussagen zu lösen. Diese empirischnormative Grauzone äußert sich in Passagen wie der folgenden: „I agree that theories of justice need empirical foundations“" (Elster 1992: 192). Wissenschaftstheoretisch bewegt Local Justice sich also eher in einer empirisch-normativen Grauzone (vgl. auch Schmidt 2000: 89ff.). Erinnerungen an das von Oncken aufgeworfene Adam-SmithProblem kommen auf (vgl. Oncken 1898). So schreibt Smith in seiner Theorie der ethischen Gefühle: „Man möge ferner auch in Betracht ziehen, daß die gegenwärtige Untersuchung nicht eine Frage des Sollens betrifft, wenn ich so sagen darf, sondern eine Frage der Tatsachen“ (Smith 1759/2004: 113). Im Nachgang zu Smiths Werken war man sich ob des fehlenden Normativitätsgehalts seiner Theorien unsicher. Auch in Elsters Rezeption wird dessen interdiziplinärer Status in manchen Schriften so weit gedehnt, dass einige Wissenschaftler behaupten, er setze sich von der sozialpsychologischen und empirischen Gerechtigkeitsforschung ${ }^{17}$ ebenso ab wie von der normativphilosophischen ${ }^{18}$. In der Literatur wird sogar vereinzelt von einem ,ambivalenten Status seiner gerechtigkeitstheoretischen Aussagen“" (Wegener 1995: 256) gesprochen, dessen Brisanz insbesondere vor dem Humeschen Diktum bzw. dem naturalistischen Fehlschluss vom Sein auf das Sollen deutlich wird. Gegen Ende der Local Justice schreibt Elster, die normative Perspektive „,would be worth exploring more fully“ (Elster 1992: 246). Elsters Selbstkritik äußert sich in der Einschätzung des Autors, seine Analyse sei „unsatisfactory in several ways“ (Elster 1992: 10):

Elster bezeichnet Walzers philosophische Argumentation jedoch als „disappointingly vague“ (Elster 1992: 14).

$17 \quad$ Unter der psychologischen und empirischen Gerechtigkeitsforschung bezeichnet man einen jüngeren Zweig der Gerechtigkeitsforschung, der v. a. psychologisch und soziologisch geprägt ist und davon ausgeht, dass es keine allgemeingültigen Gerechtigkeitsprinzipien gibt. Hingegen variieren die Gerechtigkeitsüberzeugungen je nach Individuum oder Kultur (vgl. zu einer Übersicht Ross/Miller 2002). Vgl. zur empirischen Gerechtigkeitsforschung z. B. Liebig (1997 und 2004) sowie Schmidt (2000: 55ff.).

18 Die normativ-philosophische Gerechtigkeitsforschung geht von allgemeingültigen Gerechtigkeitsprinzipien aus (vgl. zu einer Übersicht Liebig/Lengfeld 2002). 
„I am stretching my competence thinly over a large number of areas. It is not just that my treatment of the issues is selective: my knowledge is based on what may well be, in some cases, idiosyncratically chosen, unrepresentative, or dated sources" (Elster 1992: vii).

Zusammenfassend lässt sich Elsters Methode als Anbindung normativer Gerechtigkeitstheorien an die empirische Praxis oder umgekehrt als normative Reflexion empirischer Bedingungen moderner Gesellschaften benennen. Während J. Donald Moon in Elsters Theorie einen „promising start“ (Moon 1994: 181) sieht, ist Elster selbst in der Gesamteinschätzung seines Werkes skeptischer. So schreibt er: „[W] hen the circle of light expands, so does the surrounding area of darkness“" (Elster 1992: 250).

\section{Weiterentwicklungen der lokalen Gerechtigkeit}

\subsection{Kritik am Rational-Choice-Ansatz}

Elster vertritt einen Rational-Choice-Ansatz auf Basis des methodologischen Individualismus (vgl. Pies/Viebranz 2008: 190). Soziale Phänomene werden auf das Handeln - genauer: auf die Entscheidungen - einzelner Individuen zurückgeführt. Als Vertreter eines Rational-Choice-Paradigmas geht es Elster um die individuelle Entscheidung mit dem Ziel einer Nutzenmaximierung (vgl. Elster 1992: 6). Auf der anderen Seite warnt Elster vor übertriebenen Erwartungen und vor einer zu starken Vereinnahmung des methodologischen Individualismus in den Sozialwissenschaften. Er lotet damit die Grenzen des Ökonomischen aus.

„Solange die soziale Gerechtigkeitsforschung auf dem Reflexionsniveau der Entscheidungsanalyse [sic!] verharrt, kann sie Gerechtigkeit nicht anders als nutzenorientiert konzipieren“" (Wegener 1995: 257).

Auch wenn Elster selbst einen eher gemäßigten Utilitarismus (truncated utilitarianism) verfolgt: Mit dem Entscheidungsfokus des Rational-Choice-Paradigmas wird dem „Gerechtigkeitsbegriff gerade jene gesellschaftstheoretische Verankerung entzogen" (Wegener 1995: 257), die eigentlich zu leisten wäre. Um die Gesellschaft und ihre normativen Ansprüche wieder anzubinden, möchte ich choice im Folgenden in drei Schritten begründungstheoretisch (Kapitel 4.2), institutionenökonomisch (Kapitel 4.3) und vertragstheoretisch (Kapitel 4.4) - systematisch und kritisch mit Blick auf eine Wirtschafts- und Unternehmensethik weiterentwickeln.

\subsection{Choice begründungstheoretisch erweitert: Legitimität von Entscheidung}

Ein erster Kritikpunkt an Elsters Konzeption bezieht sich auf die feblende Legitimität der Entscheidungen. Allokationsentscheidungen müssen nicht nur im Lichte von Nutzenkalkülen getroffen, sondern zuvor v. a. auch begründet werden (vgl. Schmidt 2000: 135). Die Zustimmungs- und Anerkennungswürdigkeit von lokalen Gerechtigkeitsnormen fehlt also sowohl aus normativer Sicht als auch im Sinne einer faktischen Zustimmung 
aus empirischer Perspektive (vgl. auch Schmidt 2000: 135ff.). ${ }^{19}$ Wegener kritisiert Elster diesbezüglich:

„Außerhalb des Horizonts von local justice steht insbesondere die Frage nach dem Legitimationszusammenhang von Verteilungsentscheidungen (...). Inwieweit werden die Verteilungen und die Verteilungsverfahren von den Betroffenen akzeptiert, indem sie sie als gerecht empfinden und dadurch moralisch stützen?" (Wegener 1995: 257 sowie zur Vertiefung Wegener 1992: 283).

Gerechtigkeit ist in Elsters Theorie ein kontingentes Entscheidungskriterium. Zwar wird sie von den Primärakteuren als Datum in die Theorie eingespeist (Input der Theorie) und den Sekundärakteuren für ihre Entscheidung als Beschränkung (constraint) vorgegeben. Die Sekundärakteure treffen ihre Entscheidungen aber im Rahmen eines mehr oder weniger diskretionären Handlungsspielraums und führen entsprechende Spielzüge durch. Diesen Handlungsspielraum will Elster nun durch robuste Regeln einschränken, um mögliche Skandalierungen durch die Öffentlichkeit zu vermeiden. Da die Entscheidungen somit bereits „legitimiert“ sind, bleibt eine philosophische Begründung ebenso unberücksichtigt wie das faktische Gerechtigkeitsempfinden der Verteilungsempfänger, also der Tertiärakteure.

Insbesondere Diskursethiker greifen den ersten Teil der Kritik an der lokalen Gerechtigkeit auf und argumentieren für eine normative Legitimation: Nach diskursethischem Verständnis werden Normen als Output der Theorie verstanden, weil sie im Diskurs von den von einer Entscheidung Betroffenen (bei Elster wären dies die von Ausstrahlungseffekten tangierten Tertiärakteure) gemeinsam qua vernünftiger Argumentation generiert werden. Constraints im Sinne einer letatbegründeten Norm finden sich in der Diskursethik nur in der prinzipiellen Anerkennung des Anderen als Anderen und damit in der Anerkennung durch intersubjektive Reziprozität. Voraussetzung dafür wiederum ist die Rationalität ${ }^{20}$ oder - wie Ulrich in Anlehnung an Habermas formuliert - der zwanglose Zwang des besseren Argumentes (vgl. Ulrich 1993/2001: 83).

„Jede gültige Norm muß der Bedingung genügen, daß die Folgen und Nebenwirkungen, die sich aus ihrer allgemeinen Befolgung für die Befriedigung der Interessen jedes einzelnen Betroffenen voraussichtlich ergeben, von allen Betroffenen zwanglos akzeptiert werden können." (Habermas 1984: 219)

Das normative Begründungsproblem befasst sich mit der Suche nach Erklärungen und Rechtfertigungen für ethische Werturteile. Der fehlende Begründungsbezug in Elsters Theorie ist brisant, da seine Theorie in einer empirisch-normativen Grauzone zu verorten ist. Auf der einen Seite ist sie nicht-normativ, auf der anderen Seite jedoch normativ hoch relevant - man könnte auch sagen: Die fehlende Normgenerierung in der lokalen

19 Schmidt fasst unter die normative Sicht sowohl die Rechtfertigung in technischen Begriffen als auch die Rechtfertigung in ethisch-moralischen Begriffen (vgl. Schmidt 2000: 137ff.). Er orientiert sich dabei an der local justice von Elster, dass je nach Kontext auch unterschiedliche Gerechtigkeitsbegründungen geliefert werden müssen, und schlägt in diesem Zusammenhang den Begriff der adaptiven Gerechtigkeit vor (vgl. Schmidt 2000: 165ff. und Schmidt 1992b).

20 Ein Dialog heißt rational, wenn er durch „unvoreingenommene, zwanglose und nicht-persuasive Gespräche“ (Kambartel 1974: 66) erfolgt. 
Entscheidungssituation ist selbst schon eine Norm, nämlich eine solche, die aussagt, dass Entscheidungen nicht legitimiert zu werden brauchen, da sie sozusagen empirisch vorzufinden sind.

In der Diskursethik werden nicht nur universalistische Normen legitimiert (diese hat Elster nicht im Blick), sondern durchaus auch solche, die eben keinen universellen Geltungsanspruch erheben, die aber dennoch innerhalb einer Institution als handlungsleitende Normen zur Geltung gebracht werden sollen $-z$. B. Normen, die sich auf die Verantwortung von Unternehmen beispielsweise bei der Mitgestaltung der wirtschaftlichen Rahmenordnung beziehen oder die die Beachtung kartellrechtlicher und anderer wettbewerblicher Vorschriften einfordern. Sie haben selbstverständlich keinen universellen Charakter im Kantischen Sinne (vgl. Aßländer/Brink 2008). Solche Normen könnten z. B. im Rahmen deliberativer Prozesse auf ihre Akzeptanz geprüft werden (vgl. z. B. Habermas 1996 und 2001 sowie Palazzo/Scherer 2006)..21 Sie beziehen ihren Status als legitime Handlungsvorschriften aus der Zustimmung aller Betroffenen (Tertiärakteure müssen den Ausstrahlungseffekten zustimmen können). Nicht universelle, also lokale Normen, können im Rahmen deliberativer Prozesse legitimiert werden. Darüber hinaus existieren Normen, die im Kantischen Sinne als unvollkommene Pflichten gelten. Diese erscheinen zwar als verdienstvoll, sind jedoch nicht in die unmittelbare Verantwortung der Entscheidungsträger gestellt (dies wären nach Elster lokale, aber nicht begründungspflichtige Normen).

Die Verfahrensnormen sind bei Elster lokal nicht legitimiert. Wären die Prozeduren, nach denen die Inhalte bestimmt werden, für jeden Akteur einsichtig und zustimmungswürdig, dann wäre auch unkooperatives Verhalten unwahrscheinlicher: Regeln müssen also - folgt man hier z. B. Viktor Vanberg - einsichtig sein und damit den normativen Erwartungen der Akteure entsprechen. Nur dann werden sie befolgt, auch wenn sie möglicherweise gegen die eigenen Interessen und gegen den eigenen Nutzen stehen (vgl. Vanberg 1984). Die Wahrscheinlichkeit der Anerkennung sozialer Normen steigt, wenn sie den Vorstellungen des Akteurs entsprechen (vgl. Kahnemann et al. 1986 und Stolte 1987). Durch eine begründungstheoretische Erweiterung wird die dem Rational-Choice-Paradigma inhärente Gefahr eines Opportunismus bzw. strategischen Verhaltens deutlich abgeschwächt. Die nachfolgende institutionenökonomische Erweiterung verstärkt diesen Effekt.

\subsection{Choice institutionenökonomisch erweitert: Überwindung des Gefange- nendilemmas und des Prinzipal-Agent-Problems}

Die Möglichkeiten zur Auflösung von Opportunismus bzw. zur Überwindung des Gefangenendilemmas variieren von den durch Axelrod modellierten repetitiven Spielsituationen (vgl. Axelrod 1984) bis zu den zum Teil zwangsweisen Verpflichtungen zur Kooperation der Akteure. Grundsätzlich wird zwischen spielendogenen und spielexogenen Kooperationsmechanismen unterschieden (vgl. Büschges et al. 1996). Während die spielendogenen in politische Entscheidungsprozesse einbindet. Auffällig ist, dass der Subjektcharakter aufgelöst wird, hingegen Institutionen in der Gesellschaft Bedeutung erlangen. 
Kooperationsmechanismen eher auf Vereinbarungen zwischen den Akteuren basieren (self-enforcement), beziehen sich die spielexogenen Kooperationsmechanismen auf eine dritte Instanz, die über Anreize sanktioniert (third-party enforcement). ${ }^{22}$

Neben den soziologischen Vorschlägen bietet auch die Neue Institutionenökonomik Lösungen zur Überwindung so genannter Prinzipal-Agent-Probleme an. Agenturprobleme entstehen durch die Aufgabe der neoklassischen Annahme vollständiger Information. Setzt man nunmehr eine asymmetrische Informationsverteilung unter den Akteuren voraus, bieten sich drei Alternativen an, um die Agenturprobleme zu lösen: die Senkung der Informationsasymmetrien, die Harmonisierung der Ziele von Prinzipal und Agent sowie die Bildung von Vertrauen (vgl. Göbel 2002: 110ff.). Kombiniert man - in einem einfachen Prinzipal-Agent-Modell - zwei Akteure mit den Optionen vor bzw. nach Vertragsabschluss, so existieren vier Möglichkeiten, Informationsasymmetrien abzubauen: Vor Vertragsabschluss kann der Prinzipal über das so genannte screening das Unternehmen „durchleuchten“, er führt also z. B. Kontrollen durch (ex-ante, prin₹ipalseitig); beim so genannten signaling gibt der Agent von sich aus ein Signal an den Prinzipal, entweder per Gesetz (z. B. Rechenschaftsberichte), freiwillig (z. B. ethische Selbstverpflichtungen) oder über eine Zwischenform (z. B. Deutscher Corporate Governance Kodex) (ex-ante, agentseitig). Nach Vertragsabschluss können Informationsasymmetrien auf der Prinzipalseite über monitoring (ex-post, prinæipalseitig) und auf der Agentenseite über reporting (ex-post, agentseitig) reduziert werden. Eine zweite Möglichkeit zur Lösung des Agenturproblems wäre die Harmonisierung von Prinzipal- und Agenteninteresse. Auch hier könnten institutionenökonomische Maßnahmen greifen wie z. B. die Einführung von Kodizes zur Harmonisierung der Interessen durch Offenlegung von Interessenkonflikten. Schließlich kann man als dritte Maßnahme Reputationskapital aufbauen, indem in vertrauensstabilisierende Maßnahmen investiert wird.

Sämtliche drei Maßnahmen zum Abbau der Agenturprobleme betreffen also letztlich die Ausgestaltung von Verträgen (je nach Lage mal vor, mal nach Vertragsabschluss). Damit komme ich zu meinem dritten Erweiterungsvorschlag.

\subsection{Choice vertragstheoretisch erweitert: From Choice to Contract}

Elsters Reduzierung auf die einzelne Entscheidung ist aus seinen Schriften nicht immer eindeutig abzulesen. Zwar gibt er an, „to sketch a research program on the conceptions of justice held by centrally placed decision makers [sic!] and problem solvers" (Elster 1992: 238). An anderen Stellen wird jedoch deutlich, dass seine Theorie eigentlich auf mechanisms bzw. auf eine general conception abstellt (vgl. u. a. Elster 1992: 62f.). Damit ermöglicht er - neben der Legitimität (begründungstheoretische Erweiterung) und der Überwindung des Opportunismus, des Gefangenendilemmas sowie des PrinzipalAgent-Problems (institutionenökonomische Erweiterung) - eine vertragstheoretische Interpretation, insbesondere wenn man Williamsons Mechanisms of Governance in den Blick 
nimmt (vgl. Williamson 1996b). Es soll dabei ein Argument aufgenommen werden, das Williamson selbst in Anlehnung an Chester I. Barnard anführt (vgl. Barnard 1938): Governance und Regelsysteme nicht als Macht-, sondern als Konsenskomponente zu interpretieren. Hierarchie resultiert aus einem gegenseitigen Einverständnis auf Basis eines freiwillig geschlossenen Vertrages: ,authority rested on the acceptance or consent of subordinates“ (Williamson 1996a: 32). Der Vertrag wird zu einem sozialen bzw. Governance-Mechanismus, der ökonomische Transaktionen absichert. Mit Durkheim gesprochen nehmen in organisierten Gesellschaften nicht nur die vertraglich fixierten Tauschprozesse zu, sondern auch die nicht-vertraglichen Elemente (vgl. Durkheim 1988: 263). ${ }^{23}$ Sekundärakteure gehen explizite und implizite Verträge mit den Primärund den Tertiärakteuren ein: Über einen social contract etwa könnte man auch die Öffentlichkeit als vierten Akteur vertragstheoretisch berücksichtigen. Mit Bezug auf James M. Buchanan schreibt Williamson, ein solches Governance-Verständnis „moves economics in the direction of being a science of contract, as against a science of choice" (Williamson 1998: 36 [Hervorhebung A. B.] sowie vgl. insbesondere Williamson 2002). Gegeben die Annahme, ,the ultimate unit of activity (...) is a transaction" (Commons 1932: 4), müssen Transaktionen über geeignete Governancestrukturen verbunden werden (vgl. Williamson 2002: 175).

„If human actors are not only confronted with needs to adapt to the unforeseen
(by reason of bounded rationality), but are also given to strategic behavior (by
reason of opportunism), then costly contractual breakdowns (refusals of coop-
eration, maladaptation, demands for renegotiation) may be posed. In that event,
private ordering efforts to devise supportive governance structures, thereby to
mitigate prospective contractual impasses and breakdowns, have merit" (Wil-
liamson 2002: 174).

Die vertragstheoretische Perspektive kann hier nur angedeutet werden. Die Bedeutung von (kurz- und langfristigen, adaptiven etc.) Verträgen für die lokale Gerechtigkeit bedarf weiterer Forschungsbemühungen.

\section{Zum Verhältnis von lokaler Gerechtigkeit und Governanceethik}

Josef Wieland unterbreitet mit seiner Governanceethik einen Vorschlag für eine Wirtschafts- und Unternehmensethik, die nicht nur soziale Mechnismen im Sinne von Jon Elster aufgreift, sondern auch begründungstheoretische, institutionenökonomische und vertragstheoretische Erweiterungen einer Theorie lokaler Gerechtigkeit berücksichtigt, in Teilen sogar explizit herausarbeitet (vgl. Wieland 1993, 1999, 2005a/b, 2007a/b). ${ }^{24}$ Vor diesem Hintergrund soll das Verhältnis von lokaler Gerechtigkeit und

Eine weitere Erklärung könnte man daher vertragstheoretisch über unvollständige, implizite, relationale und psychologische Verträge liefern. Die unvollständigen Verträge neoklassischer Prägung können über third party enforcement geschlossen werden. Implizite, relationale und psychologische Verträge sind hingegen kommunikativ veranlagt und greifen auf andere governance structures zurück. Sie sind stärker am self enforcement orientiert (vgl. Priddat 2006: 188).

Neben den drei Erweiterungen aus dem vierten Kapitel werden im vorliegenden fünften Kapitel zugleich inhaltliche, argumentative und methodische Kritikpunkte aus dem zweiten und dritten Kapitel aufgegriffen. 
Governanceethik beleuchtet und damit die Soziologie innerhalb der Wirtschafts- und Unternehmensethik gestärkt werden.

Als Governanceethik bezeichnet Wieland grundsätzlich die Analyse bzw. Reflexion der moralischen Eigenschaften von Governancestrukturen (vgl. Wieland 1999: 46ff.), genauer versteht man darunter die

„Lehre von der komparativen Analyse der moralsensitiven Gestaltung und Kommunikation der Governancestrukturen spezifischer wirtschaftlicher Transaktionen mittels Kooperationen" (Wieland 1999: 69).

Wieland geht es ähnlich wie Elster um die Allokation von Zuteilungsgütern, bei denen nicht mehr der Markt (über den Preis), sondern die Institution (über den Sekundärakteur im Sinne Elsters) zur zentralen Steuerungsinstanz wird (vgl. auch grundsätzlich Coase 1937).

„Wird ein Vertrag gehalten, auch wenn er durch eine der beiden Parteien zu ihrem Vorteil kostenlos gebrochen werden könnte, dann alloziiert diese Partei moralische Güter, und die andere Partei erhält sie“ (Wieland 1993: 16).

Neben der Verhinderung von opportunistischem Verhalten sollen Handlungsmöglichkeiten stabilisiert und neu generiert werden: Im Zentrum steht die Schaffung von Kooperationschancen (vgl. Wieland 1999: 34). Ziel ist die ständige Verbesserung der Kooperationsfähigkeit und Kooperationsbereitschaft in einer Kooperationsökonomie, in der Wettbewerb und Kooperation vereint werden (co-opetition). ${ }^{25}$ Sind es bei Elster die ,rules that govern [sic!] the allocation of these resources“ (Elster 1992: 124), werden die sozialen Mechanismen bei Wieland als Governancemechanismen bezeichnet. Die formale Darstellung der Governanceethik lässt sich durch die nachfolgende Funktion mit vier Argumenten darstellen:

$$
\begin{gathered}
\operatorname{Tm}_{\mathrm{i}}=\mathrm{f}\left(\mathrm{aIS}_{\mathrm{i}}, \mathrm{bFI}_{\mathrm{ij}}, \mathrm{cIF}_{\mathrm{ij}}, \mathrm{dOKK} \mathrm{K}_{\mathrm{i}}\right) \\
(\mathrm{a} \ldots \mathrm{d}=-1,0,1 ; \mathrm{i}=\text { spezifische Transaktion; } \mathrm{j}=\text { spezifischer Ort })
\end{gathered}
$$

Die moralische Transaktion ergibt sich über eine spezifische Kombination der vier Argumente: IS, FI, IF und OKK. Tm steht für die moralische Dimension einer distinkten wirtschaftlichen Transaktion, die aus den nachfolgenden vier Argumenten besteht: Unter IS versteht Wieland das Regime individueller Selbstbindung oder Selbstgovernance, das in der Regel durch Tugenden, deontologische Überzeugungen o. ä. bestimmt wird. FI bezeichnet formale Institutionen wie z. B. Gesetze oder Umweltbestimmungen, IF die informalen Institutionen einer Gesellschaft oder einer Organisation wie z. B. moralische oder kulturelle Überzeugungen. Die Koordinations- und Kooperationsmechanis-

25 Zum Konzept der Co-Opetition vgl. u. a. Nalebuff/Brandenburger (1996) sowie mit Blick auf die Unternehmensethik Wieland (1999). Ausgangspunkt ist - in Anlehnung an Habermas' postnationale Konstellation - das Phänomen, dass politische Systeme national gebunden bleiben, während die Unternehmen transnationale Kooperationen eingehen (vgl. Habermas 2001). Daraus entstehen Legitimationslücken (vgl. Scherer/Palazzo 2007). Weitere Gedanken diesbezüglich finden sich in dem Buch Die Einbeziebung des Anderen (vgl. Habermas 1999). 
men einer bestimmten Organisation wie z. B. Codes of Ethics oder Codes of Conduct bilden das „Kernstück der Governanceethik“ (Wieland 2001: 17) und werden als OKK bezeichnet. Die Koeffizienten a, b, c und d geben die entsprechende Wirksamkeit an: Eine positive Wirksamkeit des Moralregimes IS z. B. wird mit 1 versehen, eine negative Wirksamkeit mit -1. Der Wert 0 deutet darauf hin, dass IS keinerlei Wirkung entfaltet. Die einzelnen Argumente werden auf eine distinkte Transaktion (i) und auf einen distinkten Kontext (j) bezogen. Damit ist die Lokalität im Ansatz von Wieland umrissen.

Optimale Moralmengen werden durch die Rahmenbedingungen bestimmt, sind definierbar und genügen Effizienzkriterien (vgl. Vanberg/Buchanan 1988 und Wieland 2007b). In Form von informellen constraints hilft Moral bei der Lösung von Gefangenendilemmasituationen der Sekundärakteure:

„Branchenkodizes sind daher ein Versuch, zwischen kollektiven (staatlichen und organisationellen) und individuellen Verträgen [sic!] ein Kontinuum von Selbstbindungsmechanismen (...) zu implementieren“"(Wieland 1993: 21).

Wieland verfolgt wie Elster einen lokalen Gerechtigkeitsansatz, da (1) die Probleme sachlich, zeitlich und sozial begrenzt sind, (2) intermediäre Entscheidungsträger vorliegen, (3) multiple Prinzipien Anwendung finden und (4) lokale Lösungen gesucht werden (vgl. Wieland 1993: 13 und 18ff.). „Die moralische Codierung kann globale Dimensionen aufweisen, während die wirtschaftliche Codierung immer lokalen [sic!] Bezug hat" (Wieland 1993: 21). Wieland bezieht sich dabei an wenigen Stellen - v. a. jüngeren Datums - explizit auf Elster, geht es doch „um die Herstellung ,lokaler Gerechtigkeit', um auf ein Forschungsprogramm von Jon Elster zu verweisen" (Wieland 1993: 23). Lokale Gerechtigkeit versteht er als „Übersetzungsprogramm differenter Systemsprachen“ (Wieland 1993: 29). Aufgrund unterschiedlicher vertraglicher Designs der Governancesysteme ergeben sich unterschiedliche lokale Gerechtigkeiten. Mit Durkheim rückt Wieland ,auf praktische Probleme zugeschnittene sanktionsbewehrte Regeln" in den Mittelpunkt seiner Theorie (Wieland 1993: 27 sowie Durkheim 1991: 15).

Die Governanceethik nimmt aber nicht nur soziologische Elemente von Elster auf, sondern integriert darüber hinaus die in Kapitel 4.2 kritisierte fehlende Legitimität aus einer eher philosophischen Perspektive. Begründungs- und Anwendungsebene werden zunächst strukturell entkoppelt, um die wirksame Governanceethik nicht aus dem Begründungs-, sondern aus dem Anwendungsdiskurs abzuleiten. So schreibt Wieland noch in einer seiner ersten Schriften zur Governanceethik:

„Ich selbst neige zu der Auffassung, dass in funktional differenzierten Gesellschaften der Vorrang der ethischen Entscheidungslogik vor der ökonomischen nur beschworen, aber nicht eingelöst werden kann“" (Wieland 1999: 83f.).

In neueren Aufsätzen hingegen greift er auf deliberative Prozesse zurück und sieht darin die Chance, die ,bisher vermisste Verbindung zwischen Begründungs- und Anwendungsdiskursen“ (Wieland 2007b: 13) nachzuliefern. Auch hier bewegt Wieland sich ähnlich wie Elster in einer empirisch-normativen Grauzone.

„Mit deliberativen Foren verbindet sich die Hoffnung eines missing links [sic!] zwischen diskursethischer Normativität und gesellschaftlicher Praxis, weil sie die demokratisch begründeten und damit gerechtfertigten Teilhabeansprüche der 
Zivilgesellschaft politisch institutionalisieren und praktisch organisieren“ (Wieland 2007b: 24).

Mit Blick auf die begründungstheoretische Erweiterung unterscheidet Wieland drei Diskurseigenschaften: Die inputorientierte Legitimität bezieht sich auf die demokratische Legitimität bzw. auf die Verfahrenslegitimität und die outputorientierte Legitimität auf die Effektivität und auf die Effizienz. Die Verfahrenslegitimität umfasst die Rechtfertigung des Prozesses (hinsichtlich der Beteiligung von Stakeholdern, der Superiorität der Legitimitätsform und der entsprechenden Einschätzung der Stakeholder selbst); Diskurseffektivität bezieht sich auf den angestrebten Output des Diskurses (hinsichtlich Inhalt, Verfahren, Implementierung) und Diskurseffizienz beschreibt die Wirtschaftlichkeit des Diskurses (hinsichtlich der Höhe der Transaktionskosten für die Durchführung des Diskurses, der Mobilisierung von Wissen und des Einsatzes der Ressourcen) (vgl. Wieland 2007b: 25).

Die Bedeutung dieser Differenzierung spiegelt sich in der Simultanität bzw. in der Interaktion der drei Diskurseigenschaften wider. Erst durch die Form der Governance wird die Legitimität zur Geltung gebracht (vgl. Wieland 2007b: 34ff. in Anlehnung an Scharpf 1997: 153) oder anders formuliert: Der Grad der Legitimität des Diskurses wird durch Diskursgovernance generiert (vgl. Wieland 2007b: 40ff.). Habermas warnt jedoch, „,nicht hinter die Legitimationsbedingungen demokratischer Selbstbestimmung zurückzufallen“ (Habermas 1982: 128, zit. nach Wieland 2007b: 19). Demokratische Legitimität als inputorientierte Legitimität ist folglich entscheidend, weil sie die formale Bedingung der prinzipiellen Anerkennung des Anderen als mündigen Gesprächspartner beinhaltet. Ideale Diskurse kombinieren die Input- und Output-Legitimität so, „,dass sie in Übereinstimmung mit den jeweiligen ,gesellschaftlichen Hintergrundüberzeugungen' (John Rawls) die Effektivität realer deliberativer Foren sicherstellen“ (Wieland 2007b: 40). Reale Diskurse hingegen sind

„lokaler Natur und charakterisiert durch eine spezifische Mischung und ein bestimmtes Verhältnis von Input-(Verfahren) und Output-(Effizienz)Legitimität, die über die Effektivitätseigenschaften der Diskurse entscheiden" (Wieland 2007b: 44f.).

Lokal gerechte Lösungen erfolgen damit über einen komplexen Abwägungsprozess als Abgleich mit den moralischen Hintergrundannahmen. Damit schließt Wieland zu Elster auf: Es gibt keine exklusiv-universalistischen Prinzipien, hingegen steht die lokale Angemessenheit und damit das Bessere anstelle des Guten im Mittelpunkt. ${ }^{26}$ Die Verbindung von Begründungs- und Anwendungsebene bzw. die begründungstheoretische Erweiterung zeigt sich in Wielands Ansatz darin, „dass diskursethische Begründungsoder Anwendungsdialoge simultane Bestandteile der informellen Institutionen einer gegebenen Gesellschaft sind und dort ihre positive Wirkung entfalten" (Wieland 2007b: 14). Zwischen den drei Diskurseigenschaften existieren Trade-off-Beziehungen: So kann z. B. die stärkere Berücksichtigung von Betroffenen (Zunahme der de-

26 Im Ergebnis gibt es daher keine „richtige“ bzw. keine „gute“ Lösung, sondern vielfach ergeben sich so genannte ,tragic choices“ (vgl. grundlegend Wieland 1999: 76 und Calabresi/Bobbit 1978). 
mokratischen Legitimität) zu einer Abnahme der Effektivität durch zunehmende Interessenkonflikte sowie zu einer Abnahme der Effizienz durch Endlosdebatten führen.

Unternehmen werden als polylinguale und polykontextuale Organisationen aufgefasst, welche lokale, d. h. auf konkrete Transaktionen bezogene Entscheidungen treffen. ${ }^{27}$ Dabei sind Argumente der unterschiedlichsten Entscheidungslogiken (moralische, rechtliche, organisatorische, wirtschaftliche etc.) gegeneinander abzuwägen. Die Legitimität der Governance - um die begründungstheoretische Erweiterung einzubeziehen - erfolgt über die „Integration polylingualer Sprachspiele“ (Wieland 2007b: 36). Ähnlich argumentiert Habermas, der die Vielfalt der Kommunikationsformen in deliberative Formen institutionalisiert (vgl. Habermas 1999, 283ff.). Soweit im Kontext von Governancestrukturen von Effizienz die Rede ist, so ist damit die Ausprägung der Adaptivität gemeint, d. h. die Anpassungsfäbigkeit bei der Abwicklung unterschiedlicher konkreter Transaktionen.

„Effizienz wird im Kontext von Governance als adaptive Effizienz [sic!] definiert, also als Vergleich von mindestens zwei Governancestrukturen hinsichtlich ihrer Anpassungsfähigkeit an Unsicherheit und Kontingenzen, die sich einstellen können bei der Abwicklung einer distinkten Transaktion“ (Wieland 1999: 47).

Eine ethische Überfrachtung durch die zu starke demokratische Legitimität führt zu idealistischen Diskursen und ist folglich zu vermeiden. Hingegen sollte man eine „angemessene und adaptive Governancestruktur" (Wieland 2007b: 36) sicherstellen. Die Adaptivität der Governance grenzt sich von dem statischen Institutionenbegriff durch die „Integration und Interaktion formaler und informaler Constraints“ (Wieland 1999: 50) ab und damit durch die Anpassungsfähigkeit von Steuerungsstrukturen an konkrete Transaktionen. Wielands Konzeption bleibt damit revisionsoffen. Auch von der Diskursethik grenzt sich der Ansatz ab, da es nicht um die Legitimität von Unternehmen bzw. deren Entscheidungen und Strategien geht, sondern um die Legitimität des gesamten Diskurses (vgl. Wieland 2007b: 36).

Wieland macht Elsters Theorie lokaler Gerechtigkeit im Rahmen seiner Governanceethik für die Wirtschafts- und Unternehmensethik anwendbar. Governance steuert die Integration und Interaktion formaler und informaler Constraints und bezeichnet damit die Adaptivität, d. h. die Anpassungsfähigkeit von Steuerungsstrukturen an konkrete lokale Transaktionen. Gerechtigkeitstheoretische Aspekte sind integraler Bestandteil von Governancestrukturen: Der systematische Ort der Gerecbtigkeit sind die lokalen Governancemechanismen selbst. Für die Soziologie ergeben sich damit interessante und weiterführende Forschungsfragen hinsichtlich der empirischen Überprüfbarkeit einer Theorie lokaler Gerechtigkeit als Governanceethik.

27 Zur Polylingualität vgl. Wieland (1999: 34f.). Wieland unterscheidet darüber hinaus zwischen globalen und lokalen Governancestrukturen. Globale Governancestrukturen, wie bspw. Staat, Markt, Rahmenordnungen oder Unternehmensverfassungen, beziehen sich auf die konstitutionellen $\mathrm{Pa}-$ rameter einer Organisation (dies entspricht Elsters Primärakteuren). Standardprozeduren, Riten und moralische Werte als Beispiele für lokale Governancestrukturen dienen der mikropolitischen Steuerung (hier finden sich Elsters Sekundärakteure). 


\section{Literatur}

Aßländer, M. S./ Brink, A. (2008): Begründung korporativer Verantwortung: Normenkonkretion als Prozess, in: Scherer, A./ Patzer, M. (Hrsg.): Betriebswirtschaftslehre und Unternehmensethik, Wiesbaden: Gabler, 103-124.

Axelrod, R. (1984): The Evolution of Cooperation, New York, NY: Basic Books.

Barnard, C. I. (1938): The Functions of the Executive, Cambridge, MA: Harvard University Press.

Büschges, G./ Abraham, M./ Funk, W. (1996): Grundzüge der Soziologie, München: Oldenbourg.

Calabresi, G./ Bobbit, P. (1978): Tragic Choices, New York, NY: Norton.

Coase, R. H. (1937): The Nature of the Firm, in: Economica, Vol. 4/No. 16, 386-405.

Commons, J. R. (1932): The Problem of Correlating Law, Economics and Ethics, in: Wisconsin Law Review, Vol. 8, 3-26.

Durkheim, É. (1988): Über soziale Arbeitsteilung: Studie über die Organisation höherer Gesellschaften, Frankfurt a. M.: Suhrkamp.

Durkheim, É. (1991): Physik der Sitten und des Rechts. Vorlesungen zur Soziologie der Moral, Frankfurt a. M.: Suhrkamp.

Elster, J. (1990): Local Justice, in: Archives Européennes de Sociologie, Vol. 31/No. 1, 117-140.

Elster, J. (1991): Local Justice: How Institutions Allocate Scarce Goods and Necessary Burdens, in: European Economic Review, Vol. 35/No. 2/3, 273-291.

Elster, J. (1992): Local Justice: How Institutions Allocate Scarce Goods and Necessary Burdens, New York, NY: Russell Sage.

Elster, J. (1995): Local Justice in America, New York, NY: Russell Sage.

Elster, J./ Herpin, N. (Hrsg.) (1992): Ethique des Choix Médicaux, Arles: Actes Sud.

Göbel, E. (2002): Neue Institutionenökonomik. Konzeptionen und betriebswirtschaftliche Anwendungen, Tübingen: UTB.

Habermas, J. (1982): Theorie des kommunikativen Handelns, Bd. 2. Zur Kritik der funktionalistischen Vernunft, Frankfurt a. M.: Suhrkamp.

Habermas, J. (1984): Vorstudien und Ergänzungen zur Theorie des kommunikativen Handelns, Frankfurt a. M.: Suhrkamp.

Habermas, J. (1996): Faktizität und Geltung, Frankfurt a. M.: Suhrkamp.

Habermas, J. (1999): Die Einbeziehung des Anderen. Studien zur politischen Theorie, Frankfurt a. M.: Suhrkamp.

Habermas, J. (2001): Die postnationale Konstellation, Frankfurt a. M.: Suhrkamp.

Hofstee, W. (1990): Allocation by Lot: A Conceptual and Empirical Analysis, in: Social Science Information, Vol. 29/No. 4, 745-763.

Kahnemann, D./ Knetsch, J. L./ Thaler, R. H. (1986): Fairness and the Assumptions of Economics, in: Journal of Business, Vol. 59/No. 4, 285-300.

Kambartel, F. (1974): Moralisches Argumentieren - Methodische Analysen zur Ethik, in: Kambartel, F. (Hrsg.): Praktische Philosophie und konstruktive Wissenschaftstheorie, Frankfurt a. M.: Suhrkamp, 54-72.

Liebig, S. (1997): Soziale Gerechtigkeitsforschung und Gerechtigkeit in Unternehmen, München: Hampp.

Liebig, S. (2004): Empirische Gerechtigkeitsforschung. Überblick über aktuelle Modelle der psychologischen und soziologischen Gerechtigkeitsforschung, ISGF-Arbeitsbericht 41.

Liebig, S./ Lengfeld, H. (2002): Gerechtigkeitsforschung als interdisziplinäres Projekt, in: Liebig, 
S./ Lengfeld, H. (Hrsg.): Interdisziplinäre Gerechtigkeitsforschung. Zur Verknüpfung empirischer und normativer Perspektiven, Frankfurt a. M.: Campus, 7-20.

Mellers, B. A. (1997): When Many Want What Only a Few Can Have, in: Journal of Behavioral Decision Making, Vol. 10/No. 4, 360-361.

Moon, J. D. (1994): Review of J. Elster (1992): Local Justice, in: Political Theory, Vol. 22/No. 1, 179-181.

Müller, H.-P. (1992): Durkheims Vision einer „gerechten“ Gesellschaft, in: Zeitschrift für Rechtssoziologie, Jg. 13/Heft 1, 16-43.

Müller, H.-P./ Wegener, B. (1995): Die Soziologie vor der Gerechtigkeit. Konturen einer soziologischen Gerechtigkeitsforschung, in: Müller, H.-P./ Wegener, B. (Hrsg.): Soziale Ungleichhit und soziale Ungerechtigkeit, Opladen: Leske + Budrich, 7-49.

Nalebuff, B./ Brandenburger, A. (1996): Coopetition - kooperativ konkurrieren. Mit der Spieltheorie zum Unternehmenserfolg, Frankfurt a. M., New York, NY: Campus.

Oncken, A. (1898): Das Adam-Smith-Problem, in: Zeitschrift für Sozialwissenschaft, Jg. 1, 25-33.

Palazzo, G./ Scherer, A. G. (2006): Corporate Legitimacy as Deliberation - A Communicative Framework, in: Journal of Business Ethics, Vol. 66/No. 1, 71-88.

Pies, I./ Viebran₹, J. (2008): Jon Elsters Theorie rationaler Bindung, in: Pies, I./ Leschke, M. (Hrsg.): Jon Elsters Theorie rationaler Bindung, Reihe: Konzepte der Gesellschaftstheorie, Bd. 14, Tübingen: Mohr Siebeck, 181-202.

Priddat, B. P. (2006): Economic Governance, in: Schuppert, G. F. (Hrsg.): GovernanceForschung. Vergewisserung über Stand und Entwicklungslinien, Baden-Baden: Nomos, 173-192.

Rawls, J. (1971): A Theory of Justice, Cambridge, MA: Belknap.

Ross, M./ Miller, D. (Eds.) (2002): The Justice Motive in Everyday Life, Cambridge: Cambridge University Press.

Scharpf, F. W. (1997): Games Real Actors Play. Actor-Centered Institutionalism in Policy Research, Boulder, CO: Westview Press.

Scherer, A. G./ Palazzo, G. (2007): Towards a Political Conception of Corporate Responsibility - Business and Society Seen from a Habermasian Perspective, in: Academy of Management Review, Vol. 32/No. 4, 1196-1120.

Schmidt, V. H. (1992a): Lokale Gerechtigkeit. Perspektiven soziologischer Gerechtigkeitsanalyse, in: Zeitschrift für Soziologie, Jg. 21/Heft 1, 3-15.

Schmidt, V. H. (1992b): Adaptive Justice: Local Distributive Justice in Sociological Perspective, in: Theory and Society, Vol. 21/No. 6, 789-816.

Schmidt, V.H. (1995): Soziologische Gerechtigkeitsanalyse als empirische Institutionenanalyse, in: Müller, H.-P./ Wegener, B. (Hrsg.): Soziale Ungleichheit und Soziale Gerechtigkeit, Opladen: Leske + Budrich, 173-194.

Schmidt, V. H. (2000): Bedingte Gerechtigkeit, Frankfurt a. M., New York, NY: Campus.

Schmidt, V. H./ Hartmann, B. K. (1997): Lokale Gerechtigkeit in Deutschland. Studien zur Verteilung knapper Bildungs-, Arbeits- und Gesundheitsgüter, Opladen: Westdeutscher Verlag.

Selten, R. (1978): The Equity Principle in Social Behavior, in: Gottinger, H./ Leinfellner, W. (Eds.): Decision Theory and Social Ethics, Dordrecht: Reidel, 289-301.

Smith, A. (1759/2004): Theorie der ethischen Gefühle, Hamburg: Meiner. 
Stolte, J. F. (1987): The Formation of Justice Norms, in: American Sociological Review, Vol. 52/No. 6, 774-784.

Ulrich, P. (1993/2001): Integrative Wirtschaftsethik. Grundlagen einer lebensdienlichen Ökonomie, 3. Aufl., Bern, Stuttgart, Wien: Haupt.

Vanberg, V. (1984): „Unsichtbare-Hand Erklärung“ und soziale Normen, in: Todt, H. (Hrsg.): Normengeleitetes Verhalten in den Sozialwissenschaften, Berlin: Duncker \& Humblot, 115-144.

Vanberg, V./ Buchanan, J. M. (1988): Rational Choice and Moral Order, in: Analyse und Kritik, Vol. 10/No. 2, 138-160.

Walzer, M. (1992): Sphären der Gerechtigkeit. Ein Plädoyer für Pluralität und Gleichheit, Frankfurt am Main: Suhrkamp.

Wegener, B. (1992): Gerechtigkeitsforschung und Legitimationsnormen, in: Zeitschrift für Soziologie, Jg. 21/Heft 4, 269-283.

Wegener, B. (1995): Auf dem Weg zur Interdisziplinarität in der sozialen Gerechtigkeitsforschung? Anmerkungen zu Scherer, Elster, Rawls und Walzer, in: Berliner Journal für Soziologie, Jg. 5/Heft 2, 251-263.

Wieland, J. (1993): Die Ethik der Wirtschaft als Problem lokaler und konstitutioneller Gerechtigkeit, in: Wieland, J. (Hrsg.): Wirtschaftsethik und Theorie der Gesellschaft, Frankfurt a. M.: Suhrkamp, 7-31.

Wieland, J. (1999): Die Ethik der Governance, Marburg: Metropolis.

Wieland, J. (2001): Eine Theorie der Governanceethik, in: Zeitschrift für Wirtschafts- und Unternehmensethik, Jg. 2/Heft 1, 8-33.

Wieland, J. (Hrsg.) (2005a): Governanceethik im Diskurs, Marburg: Metropolis.

Wieland, J. (2005b): Governanceethik und moralische Anreize, in: Beschorner, T./ Hollstein, B./ König, M./ Lee-Peuker, M.-Y./ Schumann, O. J. (Hrsg.): Wirtschafts- und Unternehmensethik. Rückblick - Ausblick - Perspektiven, München/Mering: Hampp, 251280.

Wieland, J. (Hrsg.) (2007a): Governanceethik und Diskursethik - ein zwangloser Diskurs, Marburg: Metropolis.

Wieland, J. (2007b): Idealistische, ideale und reale Diskurse. Governanceformen des Diskurses, in: Wieland, J. (Hrsg.): Governanceethik und Diskursethik - ein zwangloser Diskurs, Marburg: Metropolis, 13-58.

Williamson, O. E. (1996a): Chester Barnard and the Incipient Science of Organization, in: Williamson, O. E. (Eds.): The Mechanisms of Governance, New York: Oxford University Press, 29-53.

Williamson, O. E. (1996b): The Mechanisms of Governance, New York/Oxford: Oxford University Press.

Williamson, O. E. (1998): Transaction Cost Economics: How It Works: Where It Is Headed, in: De Economist, Vol. 146/No. 1, 23-58.

Williamson, O. E. (2002): The Theory of the Firm as Governance Structure: From Choice to Contract, in: Journal of Economic Perspectives, Vol. 16/No. 3, 171-195. 